\title{
WAVE SCATTERING AND STRESS CONCENTRATION IN A MAGNETO-ELECTRO-ELASTIC PLANE WITH A NANO-CRACK BY BOUNDARY INTEGRAL EQUATIONS
}

\author{
Yonko Stoynov ${ }^{1}$, Petia Dineva ${ }^{2}$, Tsviatko Rangelov ${ }^{3 *}$ \\ ${ }^{1}$ Faculty of Applied Mathematics and Informatics, Technical University, \\ Sofia 1000, Bulgaria \\ ${ }^{2}$ Institute of Mechanics, Bulgarian Academy of Sciences, Sofia 1113, Bulgaria \\ ${ }^{3}$ Institute of Mathematics and Informatics, Bulgarian Academy of Sciences, \\ Sofia 1113, Bulgaria
}

[Received: 9 May 2019. Accepted: 8 July 2019]

doi: 10.7546/JTAM.49.19.03.01

\begin{abstract}
Anti-plane elastodynamic problem for shear (SH) wave scattering and diffraction by a nano-crack in a magnetoelectroelastic (MEE) plane is solved herein. The presence of a nano-fracture within the MEE matrix gives rise to both wave scattering and stress concentration phenomena, the latter being responsible for fracture of the magnetoelectroelastic solid. The proposed model takes into consideration the surface/interface properties, the size of the nano-crack and the interaction between the crack, incident wave and the multifunctional MEE matrix. The method of solution is the boundary integral equation method (BIEM), which is first verified against benchmark examples and subsequently applied for numerical simulations. The BIEM formulation combines classical elastodynamic theory for the bulk solid with non-classical boundary conditions and the localized constitutive law for the matrix/nanocrack interface within the framework of the Gurtin-Murdoch theory. Also, frequency-dependent fundamental solution obtained via Radon transform for the equation of motion in the bulk MEE solid and it's derivatives are used as kernels in boundary integral equation (BIE). Results drawn from the numerical simulations reveal the degree of dependence of the dynamic stress concentration field (SCF) on the size of the nano-crack, surface and bulk material properties, coupled nature of the magnetoeletroelasticity, properties of the incident wave as frequency, wave length and wave propagation direction, dynamic interaction between the nano-crack, incident wave and coupled MEE continuum. The obtained results have applications in material science, computational fracture mechanics and nondestructive testing evaluation of multifunctional active nano-composites.
\end{abstract}

KeY wORDS: Anti-plane elastodynamics, Magnetoelectroelasticity, Nanocrack, Gurtin-Murdoch model, Wave diffraction and scattering, SCF, BIEM.

\footnotetext{
*Corresponding author e-mail: rangelov@ math.bas.bg
} 


\section{INTRODUCTION}

Magnetoelectroelastic crystals are one class of new multifunctional composites that not only consist of piezoelectric and piezomagnetic phases but also exhibit magnetoelectric coupling effects which are not present in the constituents. The magnetoelectric effect of piezoelectric / piezomagnetic composites was first reported in [1]. In the MEE crystal this effect is a coupled two field effect, in which the application of either a magnetic field or an electrical one induces an electrical polarization as well as a magnetization. A three-phase MEE composite consisting of piezoelectric and piezomagnetic phases separated by a polymer matrix have greater ductility and formability, see [2]. Due to the ability of converting one form of energy to another, these materials have a number of applications in smart or intelligent systems as intelligent sensors and actuators, damage detectors, in medical ultrasonic imaging, etc. A composite made of piezoelectric and piezomagnetic phases would be susceptible to brittle fracture because these materials are usually brittle ceramics. The electric and magnetic effects can have a significant influence on the ways with which these composite could fail by cracking. This will definitely influence the life time of devices made of them. So it is of very importance to study the fracture mechanics of MEE materials. A literature review for fracture behaviour of MEE composites with cracks at macro-level shows the following state. Static fracture problems are solved in [3-11]. Dynamic fracture behaviour of MEE composites under in-plane loading has been studied in [12-14]. SH-wave propagation in cracked MEE can be found in [15-19].

In the recent years heterogeneous by cracks and inclusions MEE nano-structures are keen of great interest because of the large magnetoelectric coupling effect in the room temperature and the small size of miniaturized multifunctional devices, see $[20,21]$. During the last few years it has been recognized that the properties of a given material might not be primarily controlled by its chemical composition, but rather by its microstructure and more so by size ranging from nanometers to micrometers. The widely discussed in the literature size effect can not be described by models in the frame-work of continuum mechanics and this demands the development of alternative models and of high-performance computational tools. In nano-structured composites, nano-scale is the most important potential advantage, and surface/interface may have significant effects on the mechanical properties of solids. Surface stress relates the variation of the excess free energy to the strain of the surface. Due to this reason the surfaces and interface solid's boundaries display different mechanical properties compared with their bulk part. One of the main thrusts of what is now known as nanoscale mechanics is the so called multi-scale approach, which aims at extending the range of classical continuum mechanics by bridging its 
basic theoretical principles with the effects observed at the molecular level. Along this direction we mention the pioneering work of $[22,23]$ who developed a general theoretical frame work to represent both surface and interface stresses. A literature review for fracture behaviour of nano-composites in the frame of Gurtin and Murdoch model shows the following state. Static fracture problems for elastic isotropic continua containing a blunt crack with crack-tips having a small curvature radius of nanometers order are considered in [24-28]. Dynamic stress field near nano-crack tip of a blunt nano-crack under incident time-harmonic $\mathrm{P}$ and SV wave in an elastic anisotropic plane is solved by BIEM in [29]. Wave scattering problems for piezoelectric composites with nano-fibers are described by wave function expansion method in [30-32]. Dynamic in-plane fracture behaviour of a nanocrack in a transversely isotropic piezoelectric plane is modelled by BIEM in [33]. The obtained results show that the surface energy significantly affects the dynamic stress and electric displacement around the nano-particle. The surface elastic effect on the dynamic mechanical stress is greater than that on the electric displacement. To the authors knowledge there are no available results for static or dynamic fracture behaviour of a nano-crack in MEE continua.

Based on the above state-of-the-art, a number of conclusions can be done:

(a) There is a certain lack of work for solution of 2D dynamic problems for elastic anisotropic materials, piezoelectric materials (PEM) and magnetoelectroelastic ones with nano-inclusions, nano-cavities and nano-cracks. The reason is that such a goal requires multidisciplinary knowledge and skills blending continuum mechanics, fracture mechanics, computational mechanics of coupled problems, material science and mathematical physics;

(b) The advantages of the BIEM are widely discussed in the literature, especially for solids with high surface-to-volume ratios, as far as the problems are described via integral equations along existing boundaries. Although well-known advantages of the BIEM for this types of problems, it's application as an effective numerical technique in nanomechanics is still limited;

(c) At macroscopic scale the crack front profile is considered to be infinite sharp and the corresponding elastic and elasto-plastic near field zones are well established in classical fracture mechanics. However, in reality the crack-tips are not ideally sharp but blunt with a curvature radius in the order of microns or nanometers. Experiments and simulations by atomic models, see [34], show that the stresses obtained via the atomic models are in a good agreement with the predictions of linear fracture mechanics except in a very small vicinity of the crack tip, where the effect of surface elastic energy should be accounted 
for. Or, the surface elasticity effect is localized at the crack-tip;

(d) There is a limited number of papers considering surface elasticity effects in cracked materials with coupled properties and the existing ones are for static loads, except [29] considering in-plane wave scattering by a nano-crack in a piezoelectric plane;

(e) To the authors knowledge there are no results for nano-cracks in the field of dynamic fracture mechanics of anisotropic magnetoelectroelastic solids.

The aim of this study is to consider the dynamic stress, electric and magnetic field near crack tip under incident time-harmonic plane SH wave in an anisotropic magnetoelectroelastic plane by BIEM taking into account the generalized surface elasticity effect in the frame of Gurtin and Murdoch model. It is considered two types of cracks: a finite line crack (Fig. 1 a) without taking into consideration the surface elasticity effects and a blunt nano-crack (Fig. 1 b) with crack root presenting by a semi-circular shape of radius $d$.

The paper is organized as follows: the statement of the problem and its reformulation by boundary integral equations is given in Sect. 2 and Sect. 3 correspondingly, a series of numerical results is presented in Sect. 4, followed by a discussion and conclusions in Sect. 5.
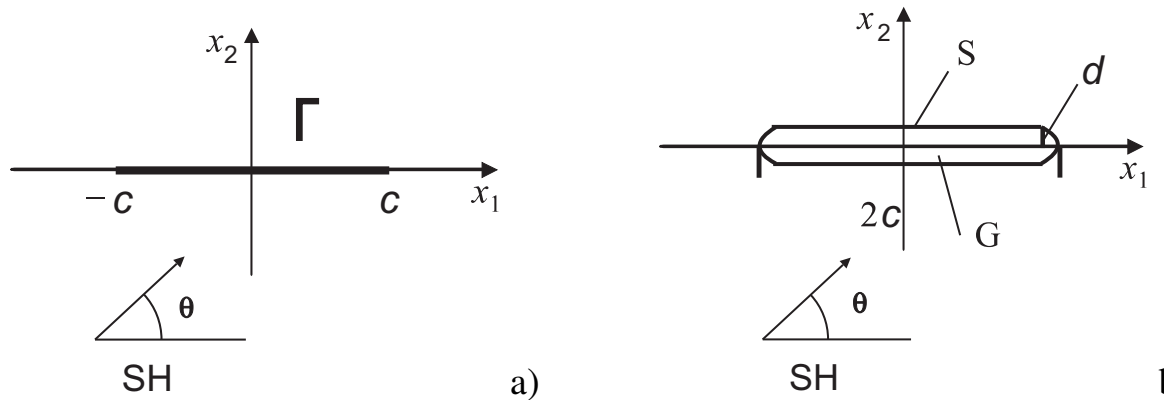

a)

$\mathrm{SH}$

b)

Fig. 1. The problem geometry: a) a line macro-crack; b) a blunt nano-crack. Cracks are subjected to incident SH- wave propagating in a transversely isotropic magneto-electro-elastic plane.

\section{Problem Statement}

Consider an infinite homogeneous transversely isotropic magnetoelectroelastic plane $x_{3}=0$ in a Cartesian coordinate system $O x_{1} x_{2} x_{3}$ with a line finite macro-crack $\Gamma=\Gamma^{+} \cup \Gamma^{-}$with half-length $2 c$ (Fig. 1 a) or a nano-crack, a domain $G$ with boundary $S$ (Fig. 1 b) subjected to incident time-harmonic with frequency $\omega$ plane 
shear $\mathrm{SH}$ wave. The $\mathrm{SH}$-wave is polarized along $\mathrm{Ox}_{3}$ axis and it propagates in the plane $x_{3}=0$ under incident angle $\theta$ with respect to the coordinate axis $O x_{1}$. Except the anti-plane mechanical time-harmonic load, in-plane electric and magnetic loads with the same frequency are applied. Assume that MEE material shows hexagonal symmetry with respect to the axis $O x_{3}$ and the poling axis is collinear with $O x_{3}$ axis. The model of the nano-crack is a blunt crack with a crack root presenting by a semicircular shape of radius $d$ and the perimeter of the crack is $|S|=2[2(c-d)+\pi d]$ with $d \ll c$, see Fig. 1b. In this case the surface effect is taken into consideration. The material properties of magnetoelectroelastic plane are: elastic stiffness coefficient $c_{44}$ piezoelectric coupled coefficient $e_{15}$, dielectric permittivity $\varepsilon_{11}$, piezomagnetic coefficient $q_{15}$, magnetoelectric coefficient $d_{11}$ and magnetic permeability $\mu_{11}$. For stable materials, the elasticity tensor, the dielectric permittivity tensor and the magnetic permeability tensor should be positive definite, see [35]. In the considered case the only non-zero field quantities all dependent on coordinates $\left(x_{1}, x_{2}\right)$ are:

- Mechanical field variables: out-of-plane mechanical displacement component $u_{3}(x, \omega)$, stress components $\sigma_{13}(x, \omega), \sigma_{23}(x, \omega)$, strain components $s_{13}(x, \omega)$, $s_{23}(x, \omega)$ and traction component $\tau_{3}(x, \omega)=\sigma_{i 3} n_{i}$, where $n_{i}$ is the outward pointing unit normal vector to the surface under consideration.

- Electrical and magnetic field variables: in-plane components of the electric field $E_{1}(x, \omega), E_{2}(x, \omega)$, electrical displacements $D_{1}(x, \omega), D_{2}(x, \omega)$, magnetic induction $B_{1}(x, \omega), B_{2}(x, \omega)$ and magnetic field $H_{1}(x, \omega), H_{2}(x, \omega)$.

The boundary value problem (BVP) we consider is defined by the governing partial differential equations and the corresponding boundary conditions given below.

\subsection{CONSTItUTIVE EQUATION}

The electromagnetic fields can be considered as quasi-static due to the reason that characteristic frequencies for electromagnetis processes are much bigger than those for elastic ones, see [36]. Then, the Maxwell equations are $D_{i, i}=0, B_{i, i}=0$, $\operatorname{rot} E=0, \operatorname{rot} H=0$ and electric and magnetic fields can be presented as gradients of scalar electric and magnetic potentials as $E_{i}=-\phi_{, i}$ and $H_{i}=-\varphi_{, i}$. The compact form of the constitutive equation in the plane $x_{3}=0$ is presented by the usage of the generalized field quantities described below, see [37]

$$
\sigma_{i K}=C_{i K J l} u_{J, l}, \quad x \in R^{2} \backslash \Gamma, \quad \text { or } x \in R^{2} \backslash G,
$$

where $i, l=1,2$, capital indexes are $K, J=3,4,5$ and it is assumed summation in repeating indexes, the generalized displacement is $u_{J}=\left(u_{3}, \phi, \varphi\right)$, where $\phi$ is 
the electric potential and $\varphi$ is the magnetic potential, the generalized stress tensor is $\sigma_{i J}=\left(\sigma_{i 3}, D_{i}, B_{i}\right)$, where $\sigma_{i 3}$ is the mechanical stress and $C_{i J K l}$ is the generalized elasticity tensor with the following components:

$$
\begin{aligned}
& C_{i 33 l}=\left\{\begin{array}{ll}
c_{44}, & i=l \\
0, & i \neq l
\end{array}, \quad C_{i 34 l}=C_{i 43 l}=\left\{\begin{array}{ll}
e_{15}, & i=l \\
0, & i \neq l
\end{array},\right.\right. \\
& C_{i 35 l}=C_{i 53 l}=\left\{\begin{array}{ll}
q_{15}, & i=l \\
0, & i \neq l
\end{array}, \quad C_{i 44 l}=\left\{\begin{array}{ll}
-\varepsilon_{11}, & i=l \\
0, & i \neq l
\end{array},\right.\right. \\
& C_{i 45 l}=C_{i 54 l}=\left\{\begin{array}{ll}
-d_{11}, & i=l \\
0, & i \neq l
\end{array}, \quad C_{i 55 l}=\left\{\begin{array}{ll}
-\mu_{11}, & i=l \\
0, & i \neq l
\end{array} .\right.\right.
\end{aligned}
$$

Note that a subscript comma denotes the partial differentiation and the summation convention over repeated indices is implied.

\subsection{KINEMATICS RELATIONS AND EQUATION OF MOTION}

Kinematics relations under assumption of small displacements and electric and magnetic field-potential relations are

$$
\begin{aligned}
& s_{i 3}(x, \omega)=u_{3, i}(x, \omega), \\
& E_{i}(x, \omega)=-\phi_{, i}(x, \omega), \\
& H_{i}(x, \omega)=-\varphi_{, i}(x, \omega), \quad i=1,2 .
\end{aligned}
$$

Equation of motion in the case there are no body force and free electric and magnetic volume charges is

$$
\sigma_{i K, i}+\rho_{K J} \omega^{2} u_{J}=0, \quad i=1,2, J, K=3,4,5,
$$

where $\rho_{Q J}= \begin{cases}\rho, & Q=J=3, \\ 0, & Q, J=4 \text { or } 5 .\end{cases}$

Note that:

(a) if $q_{15}=0$, Eq. (3) transforms to the equation of motion of transversely isotropic piezoelectric material;

(b) if $e_{15}=0$ and $q_{15}=0$, Eq. (3) transforms to the equation of motion of elastic transversely isotropic material.

The characteristic frequencies for elastic and electromagnetic processes are $f_{\mathrm{el}}=$ $10^{4} \mathrm{~Hz}$ and $f_{\mathrm{elm}}=10^{7} \mathrm{~Hz}$, respectively. Thus, if we consider dynamic loadings, with 
temporal changes corresponding to $f_{\mathrm{el}}=10^{4} \mathrm{~Hz}$, the changes of the electromagnetic fields can be assumed to be immediate, or in other words the electromagnetic fields can be considered like quasi-static, see $[8,36,38,39]$. So, the quasi-static approximation is valid and provided that the variations of the electromagnetic field with time are small enough in frequency intervals till $f_{e l}=10^{4} \mathrm{~Hz}$. Then, the Maxwell equations are $D_{i, i}=0, B_{i, i}=0, \operatorname{rot} E=0, \operatorname{rot} H=0$ and electric and magnetic fields can be presented as gradients of scalar electric and magnetic potentials as $E_{i}=-\phi_{, i}$ and $H_{i}=-\varphi_{, i}$.

\subsection{BOUNDARY CONDITIONS}

It is assumed that the crack is electrically and magnetic impermeable and in this case the electric and magnetic fields inside the both types of cracks presented in Fig. 1a,b are ignored.

Among other open problems in fracture mechanics of MEEM, the issue of how to impose the electromagnetic boundary conditions along the crack faces is a controversial one. The difficulty arises from the fact that there are not yet sufficient experimental data available, which clarify the detailed conditions at the crack faces and within the open crack. Therefore, in many cases idealized electromagnetic boundary conditions along the crack faces are employed. The impermeable assumption is based on the fact that the permittivity and permeability in the material is three orders higher than that of air or vacuum inside the crack, while in the opposite case is the permeable crack model. The physically correct boundary conditions are still an issue of debate. The aim of the authors is to show the fracture behaviour of nano-cracked MEEM for the case when the idealized electromagnetic boundary condition for impermeable crack is used. We believe that the obtained results can be useful in the discussion for the type and character of the electromagnetic boundary conditions along the crack faces.

\subsubsection{BOUNDARY CONDITION FOR MACRO-CRACK}

Boundary condition along interface $\Gamma$ between MEE matrix and the macro-crack in Fig. 1a is

$$
t_{J}=\left(\begin{array}{c}
t_{3} \\
t_{4} \\
t_{5}
\end{array}\right)=\left(\begin{array}{c}
t_{3} \\
D_{n} \\
B_{n}
\end{array}\right)=0
$$

The boundary condition along the crack line $\Gamma$ describes the assumption that the crack is free of both mechanical traction and surface electric and magnetic charge. 


\subsubsection{BOUNDARY CONDITION FOR NANO-CRACK}

The non-classical boundary condition in the frame of the $[22,23]$ model along the interface $S$, see Fig. 1b, between the MEE matrix and a blunt nano-crack is used here basing on the following assumptions:

a) The bulk material is transversely isotropic magnetoelectroelastic one describing via the constitutive equation (1);

b) The interface $S$ is considered as an infinitely thin elastic isotropic layer with own surface elastic coefficient $\mu^{S}$ and the following own constitutive equation having in mind that $s_{l 3}^{S}=\frac{1}{2} \frac{\partial u_{3}^{S}}{\partial l}$, see $[40,41]$.

$$
\sigma_{l 3}^{S}=2 c_{44}^{S} s_{l 3}^{S}=\mu^{S} \frac{\partial u_{3}^{S}}{\partial l}
$$

where $l$ is the tangential vector along the interface $S$ and $\frac{\partial u_{3}^{S}}{\partial l}$ is tangential derivative of the mechanical displacement $u_{3}^{S}$;

c) The crack is assumed to be electrically and magnetic impermeable and in this case the electric and magnetic fields inside the crack are ignored and it may be thought as a low-capacitance medium with electric and magnetic potential jumps $\Delta \phi=\phi^{+}-\phi^{-}$and $\Delta \varphi=\varphi^{+}-\varphi^{-}$. The boundary conditions for the normal component of the electric displacement and magnetic induction in this case are $D_{n}=0$ and $B_{n}=0$;

d) The infinitely thin interface layer $S$ is coherent and no atomic bonds are broken among it. Along coherent boundary which is perfect bonded, there is no slip, twist or wrinkling, the surface mechanical strain $s_{l 3}^{S}$ in tangential direction $l$ with respect to the interface boundary is equal to the associated tangential strain inside the matrix, i.e. $s_{l 3}^{S}=s_{l 3}^{M}$ and $u_{3}^{S}=u_{3}^{M}=u_{3}$.

The non-classical boundary condition in the surface elasticity theory of Gurtin and Murdoch takes into consideration a jump in the stresses as one move from the bulk material (matrix $M$ ) to the nano-crack surface $S$ due to the presence of surface stress $\sigma_{l 3}^{S}$ along $S$. It states the following relation

$$
\sigma_{n 3}^{M}(x, \omega)=t_{3}^{M}(x, \omega)=t_{3}(x, \omega)=-\frac{\partial \sigma_{l 3}^{S}}{\partial l}=-\mu^{S} \frac{\partial^{2} u_{3}}{\partial l^{2}}, x \in S
$$

Note that at zero surface elastic properties, i.e., $\mu^{S}=0$, the boundary condition (5) degenerates to the classical boundary condition $t_{3}=0$. 
The interaction of the incident time-harmonic wave with the crack line $\Gamma$ or blunt nano-crack with interface $S$ induces scattered wave field. In this case the total wave field $u_{J}$ can be written as a sum of the incident $u_{J}^{i n}$ and the scattered $u_{J}^{s c}$ wave fields. The solution for the incident wave is known and we present it here for the completeness of the text. The incident wave displacement and traction along $\Gamma$ or $S$ is as follows, see [18]:

$$
\begin{aligned}
u_{J}^{i n} & =p_{J} e^{i k\langle\eta, x\rangle}, \quad p_{3}=1, & p_{4}=A, & p_{5}=B \\
t_{3}^{i n} & =i k \frac{\operatorname{det} M}{\varepsilon_{11} \mu_{11}-d_{11}^{2}}\langle\eta, n\rangle, & t_{4}^{i n}=0, & t_{5}^{i n}=0,
\end{aligned}
$$

where $\eta=\left(\eta_{1}, \eta_{2}\right),|\eta|=1$ is the $\mathrm{SH}$ wave propagation direction and

$$
\begin{aligned}
& k=\omega \sqrt{\frac{\rho}{a}}, \quad M=\left(\begin{array}{ccc}
c_{44} & e_{15} & q_{15} \\
e_{15} & -\varepsilon_{11} & -d_{11} \\
q_{15} & -d_{11} & -\mu_{11}
\end{array}\right), \quad a=\frac{\operatorname{det} M}{\mu_{11} \varepsilon_{11}-d_{11}^{2}}, \\
& A=\frac{\mu_{11} e_{15}-q_{15} d_{11}}{\mu_{11} \varepsilon_{11}-d_{11}^{2}}, \quad B=\frac{q_{15} \varepsilon_{11}-d_{11} e_{15}}{\mu_{11} \varepsilon_{11}-d_{11}^{2}} .
\end{aligned}
$$

Finally, the solution for the total wave field of the defined BVP satisfies the governing Eq. (3), the boundary condition (4) for the line crack in Fig. 1 a, or (5) for the blunt nano-crack in Fig. $1 \mathrm{~b}$ and at infinite the Sommerfeld's radiation condition for the scattered wave is satisfied.

\section{BIEM Formulation OF THE PROBLEM}

The BVP defined above can be reformulated (see [18]) using a set of traction based non-hypersingular boundary integral equations along the line $\Gamma$ (in the case of a line macro-crack) and along the interface $S$ (in the case of a blunt nano-crack) derived via combine usage of the two-state conservation integral of elastodynamics and the frequency-dependent fundamental solution of Eq. (3).

\subsection{MACRO-CRACK}

The following integro-differential equation along the line $\Gamma$ (Fig. 1 a) with respect to the unknown crack opening displacement (COD) of the scattered wave field defined as $\Delta u_{J}^{s c}=\left.u_{J}^{s c}\right|_{\Gamma^{+}}-\left.u_{J}^{s c}\right|_{\Gamma^{-}}$, describes the formulated BVP in the case of the line macro-crack:

(8) $t_{J}^{i n}(x, \omega)=C_{i J K l} n_{i}(x) \int_{\Gamma}\left[\left(\sigma_{\eta P K}^{*}(x, \xi, \omega) \Delta u_{P, \eta}^{s c}(\xi, \omega)\right.\right.$

$\left.\left.-\rho_{Q P} \omega^{2} u_{Q K}^{*}(x, \xi, \omega) \Delta u_{P}^{s c}\right) \delta_{\lambda l}-\sigma_{\lambda P K}^{*}(x, \xi, \omega) \Delta u_{P, l}^{s c}(\xi, \omega)\right] n_{\lambda}(\xi) d \xi, \quad x \in \Gamma$, 
where $x=\left(x_{1}, x_{2}\right)$ and $\xi=\left(\xi_{1}, \xi_{2}\right)$ are the position vectors of the source and field points, $u_{Q K}^{*}$ is the fundamental solution of Eq. (3) and its stress is $\sigma_{i J Q}^{*}=$ $C_{i J M l} u_{M Q, l}^{*}, \delta_{\lambda l}$ is the Kronecker symbol. The fundamental solution $u_{Q K}^{*}$ is derived by the usage of Radon transform and the details can be found in [18]. The solution of the Eq. (8) is obtained after application of the discretization and collocation procedures discussed in details in [8].

\subsection{BLUNT NANO-CARCK}

In here we use integro-differential equation with respect to the scattered wave displacement along the interface $S$ between the MEE matrix and a blunt nano-crack presented in Fig. 1 b

(9) $\begin{aligned} & \gamma_{R J}\left(t_{R}^{i n}(x, \omega)-t_{R}^{M}(x, \omega)\right)=C_{i J K l} n_{i}(x) \int_{S}\left[\left(\sigma_{\eta P K}^{*}(x, \xi, \omega) u_{P, \eta}^{s c}(\xi, \omega)\right.\right. \\ & \left.\left.-\rho_{Q P} \omega^{2} u_{Q K}^{*}(x, \xi, \omega) u_{P}^{s c}\right) \delta_{\lambda l}-\sigma_{\lambda P K}^{*}(x, \xi, \omega) u_{P, l}^{s c}(\xi, \omega)\right] n_{\lambda}(\xi) d \xi, \quad x \in S,\end{aligned}$

$\gamma_{R J}$ is the jump term depending on the local geometry at the source point $x$ and functions in Eq. (9) are defined in Section 3.1. After discretization and collocation procedure applied to Eq. (9) where we use the non-classical boundary condition (Eq. (5)) the obtained solution takes into consideration the discussed above effects of the surface elasticity via Gurtin - Murdoch model.

\subsection{GSIF AND GSCF EVALUATION}

Once the solution of the boundary integral equation (BIE) (8) or (9) is known for a fixed frequency $\omega$, the displacement and traction at any observer point in the MEE plane can be obtained via the known representation formulae.

The computation of the generalized stress intensity factor (GSIF) can be done by the usage of the well-known traction formulae. If we consider a line crack (Fig. 1 a) the traction formulae give:

$$
\begin{aligned}
& K_{I I I}=\lim _{x_{1} \rightarrow \pm c} t_{3} \sqrt{2 \pi\left(x_{1} \mp c\right)}, \\
& K_{D}=\lim _{x_{1} \rightarrow \pm c} t_{4} \sqrt{2 \pi\left(x_{1} \mp c\right)}, \\
& K_{B}=\lim _{x_{1} \rightarrow \pm c} t_{5} \sqrt{2 \pi\left(x_{1} \mp c\right)} .
\end{aligned}
$$

The following formulae for the electric $E_{2}$ and magnetic $H_{2}$ field components respectively are obtained in the case of normal incident wave

$$
E_{2}=\frac{\operatorname{det} M_{2}}{\operatorname{det} M}, H_{2}=\frac{\operatorname{det} M_{3}}{\operatorname{det} M},
$$


where

$$
\begin{gathered}
M=\left(\begin{array}{ccc}
c_{44} & e_{15} & q_{15} \\
e_{15} & -\varepsilon_{11} & -d_{11} \\
q_{15} & -d_{11} & -\mu_{11}
\end{array}\right), \\
M_{2}=\left(\begin{array}{ccc}
c_{44} & t_{3} & -q_{15} \\
e_{15} & t_{4} & d_{11} \\
q_{15} & t_{5} & \mu_{11}
\end{array}\right), \quad M_{3}=\left(\begin{array}{ccc}
c_{44} & -e_{15} & t_{3} \\
e_{15} & \varepsilon_{11} & t_{4} \\
q_{15} & d_{11} & t_{5}
\end{array}\right) .
\end{gathered}
$$

The electric field intensity factor $K_{E}$ and the magnetic field intensity factor $K_{H}$ are calculated with formulae

$$
\begin{aligned}
& K_{E}=\lim _{x_{1} \rightarrow \pm c} E_{2} \sqrt{2 \pi\left(x_{1} \mp c\right)}, \\
& K_{H}=\lim _{x_{1} \rightarrow \pm c} H_{2} \sqrt{2 \pi\left(x_{1} \mp c\right)}
\end{aligned}
$$

The normalized GSIFs are

$$
K_{I I I}^{*}=K_{I I I}\left(t_{3}^{i n} \sqrt{\pi c}\right)^{-1}, \quad K_{E}^{*}=K_{E}\left(t_{3}^{i n} \sqrt{\pi c}\right)^{-1}, \quad K_{H}^{*}=K_{H}\left(t_{3}^{i n} \sqrt{\pi c}\right)^{-1}
$$

The computation of the generalized normalized stress concentration factor (GSCF) close to the interface $S$ of a blunt nano-crack (Fig. 1 b) at point $\left( \pm x_{1}, 0\right)$ is proceed with the formulae

$$
\begin{aligned}
& F_{I I I}^{*}\left(\left(x_{1}, 0\right), \omega\right)=\frac{t_{3}\left(\left(x_{1}, 0\right), \omega\right)}{t_{3}^{i n}\left(\left(x_{1}, 0\right), \omega\right)} \sqrt{2 \pi\left(x_{1} \mp c\right)}, \quad\left|x_{1}\right|>c, \\
& F_{E}^{*}\left(\left(x_{1}, 0\right), \omega\right)=\frac{E_{n}\left(\left(x_{1}, 0\right), \omega\right)}{t_{3}^{i n}\left(\left(x_{1}, 0\right), \omega\right)} \sqrt{2 \pi\left(x_{1} \mp c\right)}, \quad\left|x_{1}\right|>c, \\
& F_{H}^{*}\left(\left(x_{1}, 0\right), \omega\right)=\frac{H_{n}\left(\left(x_{1}, 0\right), \omega\right)}{t_{3}^{i n}\left(\left(x_{1}, 0\right), \omega\right)} \sqrt{2 \pi\left(x_{1} \mp c\right)},
\end{aligned}
$$

For the considered homogeneous MEE, using Eqs. (3), (4), (5) and (7) it can be proved that

$$
K_{E}^{*}=A K_{I I I}^{*}, \quad K_{H}^{*}=B K_{I I I} ; \quad F_{E}^{*}=A F_{I I I}, \quad F_{H}^{*}=B F_{I I I} .
$$

Equation (14) means that in the numerical examples it is enough to plot only $K_{I I I}^{*}$ and $F_{I I I}^{*}$. 


\section{Numerical Procedure ANd Numerical Results}

The non-hypersingular traction BIEs (8) is collocated along one side of the line crack $\Gamma$ using displacement jumps, i.e., COD as unknowns in the case of macro-crack. In the case of the blunt nano-crack the collocation is on both sides of the interface $S$. The generalized field variables are approximated with parabolic shape functions which satisfy Hölder continuity at least at the collocation points and show an asymptotic $O(\sqrt{r})$ - COD behaviour near the crack tips. Quarter-point boundary elements (QP-BE) are implemented in a quadratic boundary element discretization. The disadvantage of the standard quadratic approximation regarding the smoothness at all irregular points is overcome by the shifted point method, see [8]. After discretization the obtained integrals are at least Cauchy principal value integrals. The regular integrals are computed employing the Gaussian quadrature scheme for one-dimensional integrals and Monte Carlo integration scheme for two-dimensional integrals. All integrals with singular kernels are solved analytically in the small neighbourhood of the field point, using the approximation of the fundamental solution for a small argument. After discretization of the non-hypersingular traction BIEs and satisfying boundary conditions on the macro-crack or nano-crack, an algebraic system of equations in respect to the unknowns is obtained and solved. The program codes basing on Mathematica 6 have been created following the above described procedure.

The following discretization mesh is applied in the numerical scheme

(a) 5 boundary elements are used for the line crack $\Gamma$ with half-length $c$, where the first and the fifth one are quarter-point crack-tip boundary elements with length $0.15 c$ and $c=5 \times 10^{-9} \mathrm{~m}$;

(b) 10 ordinary boundary elements are used for the blunt nano-crack with boundary $S$ with length $|S|=2.0428 \times 10^{-8} \mathrm{~m}, 8$ along $S^{-} \cup S^{+}$and 2 along semi-circles $S^{l}, S^{r}$ with a fixed radius $d=0.0375 c$.

Three types of materials are considered

Material I: piezoelectric material $\mathrm{BaTiO}_{3}$ with elastic stiffness $c_{44}^{0}=43(\mathrm{GPa})$, dielectric permittivity $\varepsilon_{11}^{0}=11.2 \times 10^{-10}\left(\mathrm{C}^{2} / \mathrm{Nm}^{2}\right)$, piezoelectric coefficient $e_{15}^{0}=11.6\left(\mathrm{C} / \mathrm{m}^{2}\right)$ and density $\rho^{0}=5.8 \times 10^{3}\left(\mathrm{~kg} / \mathrm{m}^{3}\right)$;

Material 2: piezomagnetic material $\mathrm{CoFe}_{2} \mathrm{O}_{4}$ with elastic stiffness $c_{44}^{0}=45.3(\mathrm{GPa})$, dielectric permittivity $\varepsilon_{11}^{0}=0.08 \times 10^{-10}\left(\mathrm{C}^{2} / \mathrm{Nm}^{2}\right)$, magnetic permeability $\mu_{11}^{0}=-590 \times 10^{-6}\left(N s^{2} / C^{2}\right)$, piezomagnetic coefficient $q_{15}^{0}=550(\mathrm{~N} / \mathrm{Am})$ and density $\rho^{0}=7.5 \times 10^{3}\left(\mathrm{~kg} / \mathrm{m}^{3}\right)$;

Material 3: magnetoelectroelastic composite $\mathrm{BaTiO}_{3} / \mathrm{CoFe}_{2} \mathrm{O}_{4}$. It is assumed that the material constants $k^{c}$ of the composite made of $\mathrm{BaTiO}_{3}$ as an inclusion 
with material constants $k^{i}$ and $\mathrm{CoFe}_{2} \mathrm{O}_{4}$ as a matrix with material constants $k^{m}$ are defined as $k^{c}=0.5\left(k^{i}+k^{m}\right)$, see [10]. The magnetoelectric constant $d_{11}^{0}=5.2 \times 10^{-12}(\mathrm{Ns} / \mathrm{VC})$ is chosen based on the micro-mechanical model, proposed in [15].

It is used dimensionless surface parameter defined as $s=\mu^{S} /\left(2 c_{44} d d_{s}\right)$. In the numerical results we get the values of $\mu^{S}$ from the literature, usually determined by the atomic simulations $([9,42])$ and they are in the neighbourhood of $\pm 10 \mathrm{~N} / \mathrm{m}$, where negative values are also possible. Note that the case $s=0$, i.e. crack without surface effect, corresponds to the line crack $\Gamma$ presented in Fig. 1 a.

To the authors best knowledge, there are no available results in the literature for MEE material with a nano-crack subjected to dynamic loads. In order to verify the present numerical scheme we will compare our BIEM solutions for a blunt nanocrack (Figure 1b) at zero surface elasticity parameter $s=0$ with the authors' and available in the literature results for a straight macro-crack (Fig. 1 a). In the discussed below test example is applied the numerical scheme proposed here for a coupled magnetoelectroelastic material. In Fig. 2 is presented SIF-III versus normalized frequency $\Omega=c \omega \sqrt{c_{44} / \rho}$ of normal incident SH wave propagating in a magnetoelectroelastic plane of $\mathrm{BaTiO}_{3} / \mathrm{CoFe}_{2} \mathrm{O}_{4}$. A comparison is presented between the BIEM solutions obtained for a line crack (Fig. 1 a) in [18] and the current BIEM solutions for a blunt nano-crack (Fig. $1 \mathrm{~b}$ ) at surface parameter $s=0$.

The results obtained in Fig. 2 demonstrate the high accuracy and good convergence of the BIEM solutions at considered frequency interval.

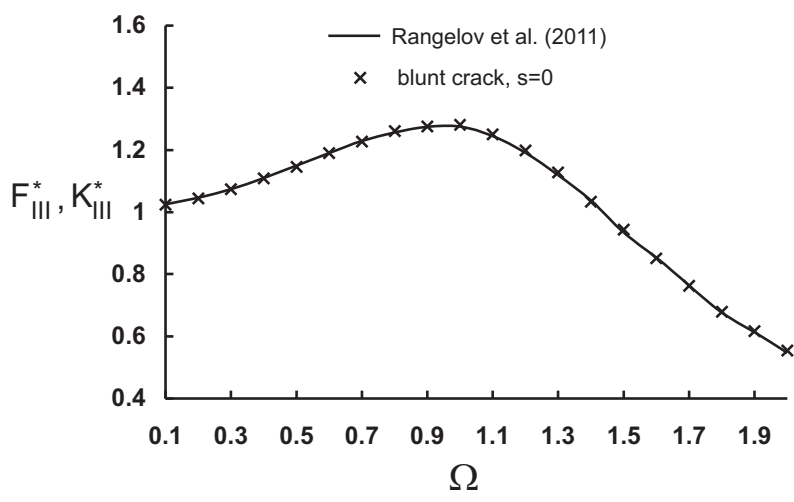

Fig. 2. Normalized SIF $K_{I I I}^{*}$ and normalized SCF $F_{I I I}^{*}$ versus normalized frequency $\Omega$ of normal incident $\mathrm{SH}$ wave propagating in magnetoelectroelastic plane of $\mathrm{BaTiO}_{3} / \mathrm{CoFe}_{2} \mathrm{O}_{4}$. Comparison between solutions for a line crack (Fig. 1 a) and a blunt nano-crack (Fig. 1 b) at surface parameter $s=0$. 
In the following, a set of numerical results for a wave loaded blunt nano-crack is presented highlighting the dependence of the dynamic frequency stress concentration field on key parameters as the wave characteristics, the bulk material properties, the surface material properties, the surface elasticity effect, the crack-size, magnetoelectro-elastic coupling and the wave-crack-MEE material interaction.

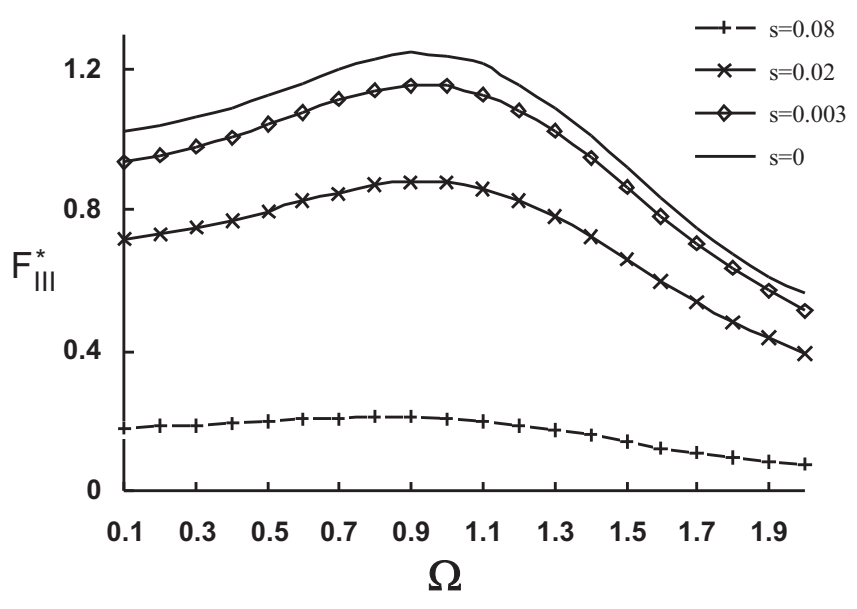

Fig. 3. Normalized SCF $F_{I I I}^{*}$ versus normalized frequency $\Omega$ of normal incident SH wave propagating in a plane of Material $1\left(\mathrm{BaTiO}_{3}\right)$ containing a blunt nano-crack for different values of undimensional surface parameter $s=0,0.003,0.08,0.02$.

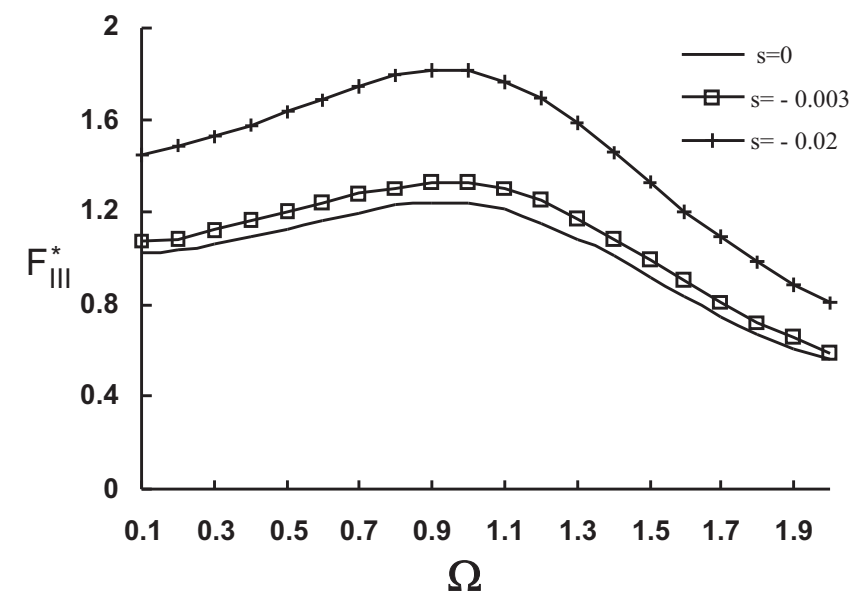

Fig. 4. Normalized SCF $F_{I I I}^{*}$ versus normalized frequency $\Omega$ of normal incident SH wave propagating in a plane of Material $1\left(\mathrm{BaTiO}_{3}\right)$ containing a blunt nano-crack for different values of undimensional surface parameter $s=0,-0.003,-0.02$. 


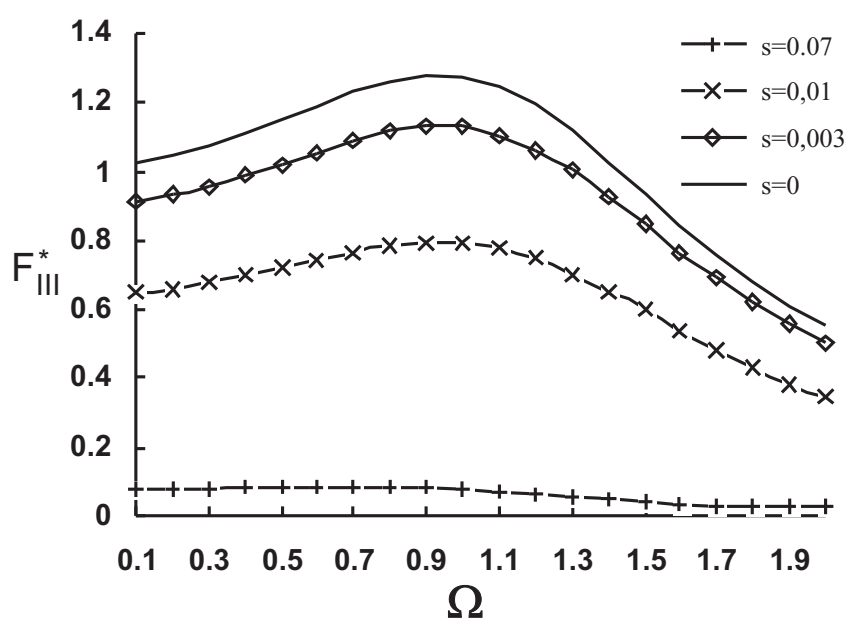

Fig. 5. Normalized SCF $F_{I I I}^{*}$ versus normalized frequency $\Omega$ of normal incident SH wave propagating in a plane of Material $2\left(\mathrm{CoFe}_{2} \mathrm{O}_{4}\right)$ containing a blunt nano-crack for different values of undimensional surface parameter $s=0,0.003,0.01,0.07$.

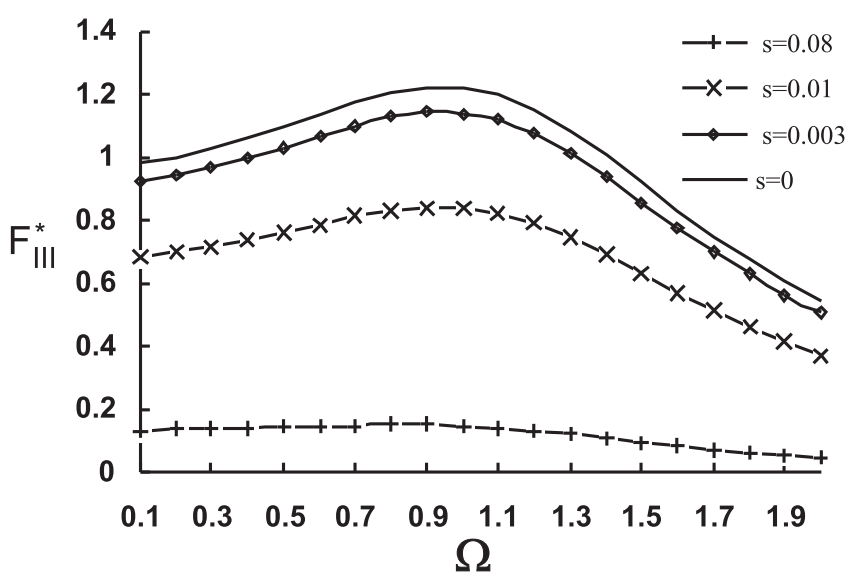

Fig. 6. Normalized SCF $F_{I I I}^{*}$ versus normalized frequency $\Omega$ of normal incident SH wave propagating in a plane of Material $3\left(\mathrm{BaTiO}_{3} / \mathrm{CoFe}_{2} \mathrm{O}_{4}\right)$ containing a blunt nano-crack for different values of undimensional surface parameter $s=0,0.003,0.01,0.08$.

Figures 3-6 draw the absolute value of SCF $F_{I I I}^{*}$ versus normalized frequency $\Omega$ of normal incident SH wave propagating in a plane of Material 1, Material 2 and Material 3 correspondingly, and containing a blunt nano-crack at different values of the undimensional surface parameter $s$. Figures $7-9$ present the absolute value of SCF $F_{I I I}^{*}$ at observer point $x_{1}=c .10^{p-6}, p=1, \ldots, 7$ for normal incident SH wave propagating with a fixed frequency $\Omega=0.3$ in a plane of Material 1, Material 2 and 
Material 3 correspondingly, and containing a blunt nano-crack at different values of undimensional surface parameter $s$.

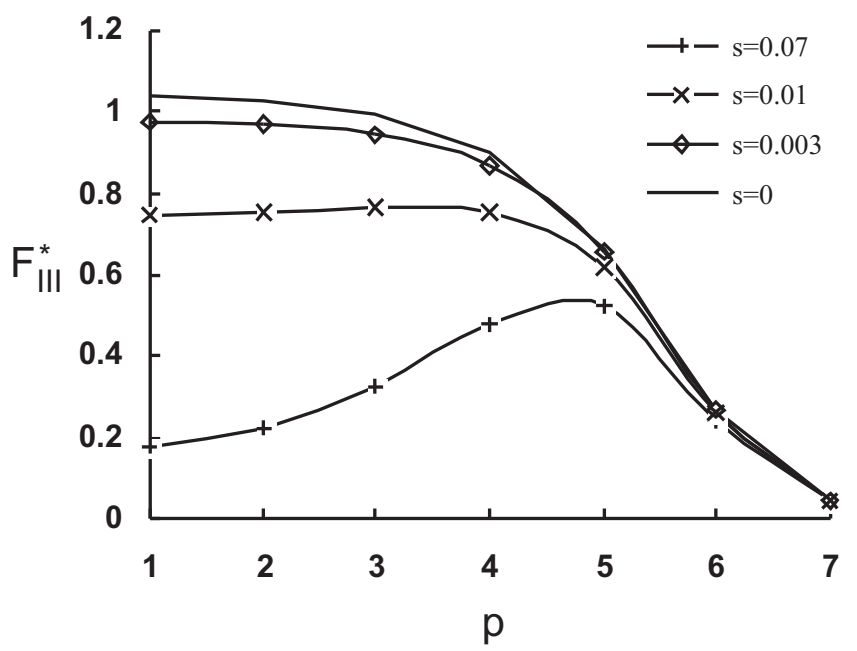

Fig. 7. Normalized SCF $F_{I I I}^{*}$ at observer point $x_{1}=c .10^{p-6}, p=1, \ldots, 7$ of normal incident SH wave propagating with normalized frequency $\Omega=0.3$ in a plane of Material 1 $\left(\mathrm{BaTiO}_{3}\right)$ containing a blunt nano-crack for different values of undimensional surface parameter $s=0,0.003,0.01,0.07$.

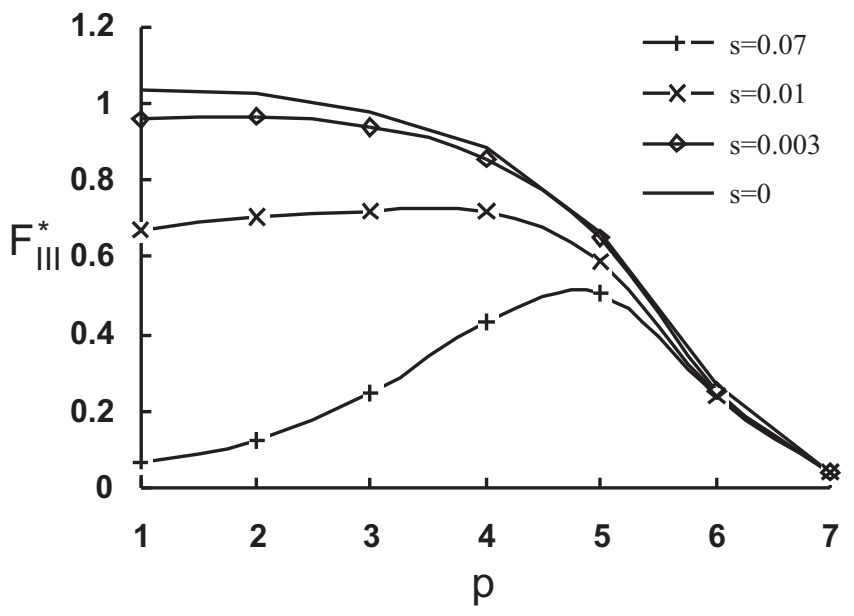

Fig. 8. Normalized SCF $F_{I I I}^{*}$ at observer point $x_{1}=c \cdot 10^{p-6}, p=1, \ldots, 7$ of normal incident SH wave propagating with normalized frequency $\Omega=0.3$ in a plane of Material $2\left(\mathrm{CoFe}_{2} \mathrm{O}_{4}\right)$ containing a blunt nano-crack for different values of undimensional surface parameter $s=0,0.003,0.01,0.07$. 


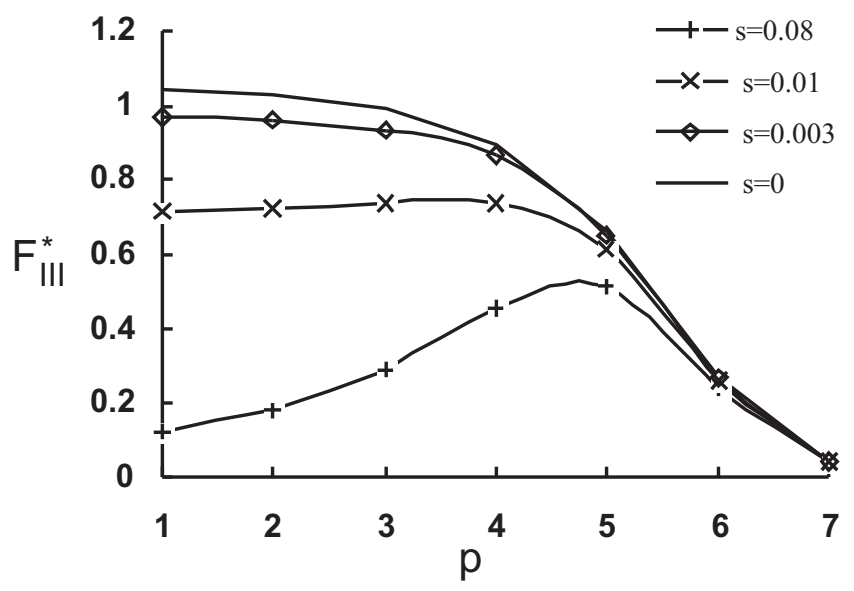

Fig. 9. Normalized SCF $F_{I I I}^{*}$ at observer point $x_{1}=c \cdot 10^{p-6}, p=1, \ldots, 7$ of normal incident $\mathrm{SH}$ wave propagating with normalized frequency $\Omega=0.3$ in a plane of Material $3\left(\mathrm{BaTiO}_{3} / \mathrm{CoFe}_{2} \mathrm{O}_{4}\right)$ containing a blunt nano-crack for different values of undimensional surface parameter $s=0,0.003,0.01,0.08$.

The obtained results illustrate clear that the stress concentration field near the crack-tip decreases with increasing of the positive surface parameter $s$ and increases with increasing of $s$ at its negative values, see Fig. 4. The surface effects can cause a strong transformation (increase or decrease) of the stress concentration fields and this effect is governing via the surface elasticity properties. In the case of $s=0$, the obtained solution recovers those for the macro-crack. The obtained results reveal the sensitivity of the SCF to the frequency of the dynamic load, to the specific surface properties and to the size of the blunt cracks root curvature. The above analyses demonstrate and confirm the suggestion that surface elasticity has a considerable impact on the near-tip fields of the crack in MEE composite materials.

\section{CONCLUSION}

A 2D fracture analysis of a blunt nano-crack in an infinite transversely isotropic magnetoeletroelastic plane under incident $\mathrm{SH}$ wave by non-hypersingular traction BIEM in conjunction with the surface elasticity model of Gurtin and Murdoch is presented. The developed and verified numerical scheme is based on: (a) the fundamental solution of dynamic equilibrium equation for transversely isotropic MEE material derived analytically by the use of Radon transform; (b) the non-classical boundary condition in the frame of Gurtin and Murdoch model along the negligibly thin interface surface expressing a jump of generalized stresses from the matrix to the nano-blunt crack due to the presence of surface stress field. A parametric study for local dynamic stress concentration reveals that the wave-nano-crack interaction in 
anisotropic MEE continua depends on the combined influence of the following key parameters: (a) properties of the incident wave; (b) the ratio of the wave length and the crack length; (c) the type of surface and bulk material properties; (d) the magnetoelectro-mechanical coupling and (e) the size of the blunt crack root curvature. The strong influence of the size-effect of nano-cracks to the dynamic fracture behaviour of cracked MEE materials can significantly affect its overall effective dynamic fracture behaviour. The obtained results demonstrate clearly that the MEE composite materials containing nanoscale cracks possess unique fracture behaviour different from those at macroscale.

\section{ACKNOWLEDGEMENT}

The second and third authors are grateful for support provided under research grant IB-RA2014-178-EnTranEmiss from the Federal Ministry of Education Research in Germany. This paper is partially supported by the National Scientific Program "Information and Communication Technologies for a Single Digital Market in Science, Education and Security (ICTinSES)", contract No DO1-205/23.11.2018, financed by the Ministry of Education and Science in Bulgaria and also by the project BG05M2OP0011.002, MIRACle 2019-2023.

\section{REFERENCES}

[1] J. van Suchteln (1972) Product Properties: A New Application of Composite Materials. Philips Research Reports 27 28-37.

[2] L. Jaesang, G. James, I.V. Boyd, C.L. Dimitris (2005) Effective Properties of Three-Phase Electro-Magnetoelastic Composites. International Journal of Engineering Science $\mathbf{4 3}$ 790-825.

[3] C.P. Spyropoulos, G.C. SiH, Z.F. Song (2003) Magnetoelectroelastic Composite with Poling Parallel to Plane of Line Crack under Out-Of-Plane Deformation. Theoretical and Applied Fracture Mechanics 40 281-289.

[4] B.L. WAng, Y.W. MaI (2003) Crack-Tip Field in Piezoelectric/Piezomagnetic Media. European Journal of Mechanics endash; A/Solids 22 591-602.

[5] B.L. WANG, Y.W. MAI (2004) Fracture of Piezoelectromagnetic Materials. Mechanics Research Communications 31 65-73.

[6] B.L. Wang, Y.W. MaI (2007) Applicability of the Crack-Face Electromagnetic Boundary Conditions for Fracture of Magnetoelectroelastic Materials. International Journal of Solids and Structures 44 387-398.

[7] F. Garcia-Sanchez, R. Rojas-Diaz, A. Saez, C. Zhang (2007) Fracture of Magnetoelectroelastic Composite Materials Using Boundary Element Method (BEM). Theoretical and Applied Fracture Mechanics 47 192-204. 
[8] P. Dineva, D. Gross, R. Müller, T. Rangelov (2014) "Dynamic Fracture of Piezoelectric Materials. Solutions of Time-Harmonic Problems via BIEM”. Springer Science \& Business Media.

[9] V.B. Shenoy (2002) Size-Dependent Rigidities of Nanosized Torsional Elements. International Journal of Solids and Structures 39 4039-4052.

[10] R.K.L. Su, H.Y. Sun (2003) Numerical Solution of Two-Dimensional Anisotropic Crack Problems. International Journal of Solids and Structures 40 4615-4635.

[11] C.C. MA, J.M. LEE (2009) Theoretical Analysis of In-Plane Problem in Functionally Graded Nonhomogeneous Magnetoelectroelastic Bimaterials. International Journal of Solids and Structures 46 4208-4220.

[12] R. Rojas-Diaz, A. Saez, F. Garcia-SAnchez, C. Zhang (2008) Time-Harmonic Greens Functions for Anisotropic Magnetoelectroelasticity. emphInternational Journal of Solids and Structures 45 144-158.

[13] R. Rojas-Diaz, A. Saez, F. Garcia-Sanchez, C. Zhang (2009) Dynamic Crack Interactions in Magnetoelectroelastic Composite Materials. International Journal of Fracture 157(1-2)119-130.

[14] R. Rojas-Diaz, A. Saez, F. Garcia-Sanchez, C. Zhang (2010) Analysis of Cracked Magnetoelectroelastic Composites under Time-Harmonic Loading. International Journal of Solids and Structures 47 71-80.

[15] X.F. Li (2005) Dynamic Analysis of a Cracked Magneto-Electro-Elastic Medium under Anti-Plane Mechanical and In-Plane Electric And Magnetic Impacts. International Journal of Solids and Structures 42 3185-3205.

[16] R.K. SU, W.J. Feng, J. LiU (2007) Transient Response of Interface Cracks between Dissimilar Magneto-Electro-Elastic Strips under Out-Of-Plane Mechanical and Inplane Magneto-Electrical Impact Loads. Composite Structures 78 119-128.

[17] Z. Zhou, B. WANG (2006) Dynamic Behaviour of Two Parallel Symmetry Cracks in Magneto-Electro-Elastic Composites under Harmonic Anti-Plane Waves. Applied Mathematics and Mechanics 27 583-591.

[18] T. Rangelov, Y. Stoynov, P. Dineva (2011) Dynamic Fracture Behavior of Functionally Graded Magnetoelectroelastic Solids by BIEM. International Journal of Solids and Structures 48 2987-2999.

[19] Z. Zhou, B. WANG (2005) Scattering of Harmonic Anti-Plane Shear Waves by an Interface Crack in Magneto-Electro-Elastic Composites. Applied Mathematics and Mechanics 26 17-26.

[20] C. W. Nan, M.I. Bichurin, S.X. Dong, D. Viehland, G. Srinivasan (2008). Multiferroic Magnetoelectric Composites: Historical Perspective, Status, and Future Directions. Journal of Applied Physics 103031101.

[21] T. Brintlinger, S. Lim, K. BAloch, P. Alexander, Y. Qi, J. BARry, J. MelNGAilis, L. Salamanca-Riba, I. TAKeuchi, J.J. Cumings (2010) In Situ Observation of Reversible Nanomagnetic Switching Induced by Electric Fields. Nano Letters 10 12-19. 
[22] M.E. GuRTin, A.I. Murdoch (1975) A Continuum Theory of Elastic Material Surfaces. Archive for Rational Mechanics and Analysis 57 291-323.

[23] M.E. Gurtin, A.I. Murdoch (1978) Surface Stress in Solids. International Journal of Solids and Structures 14 431-440.

[24] G.F. WANG, Y. Li (2013) Influence of Surface Tension on Mode I Crack Tip Field. Engineering Fracture Mechanics 109 290-301.

[25] G.F. Wang, X.Q. Feng, T.J. Wang, W. GaO (2008) Surface Effects on the NearTip Stresses for Mode-I and Mode-III Cracks. ASME, Journal of Applied Mechanics 75 011001.

[26] X. L. Fu, G.F. Wang, X.Q. Feng (2008) Surface Effects on the Near-Tip Stress Felds of A Mode-II Crack. International Journal of Fracture 151 95-106.

[27] X.L. Fu, G.F. WAng, X.Q. Feng (2010) Surface Effects on Mode-I Crack-Tip Fields: A Numerical Study. Engineering Fracture Mechanics 77 1048-1057.

[28] X.L. Fu, G.F. WANG, X.Q. Feng (2011) Effects of Surface Elasticity on Mixed-Mode Fracture. International Journal of Applied Mechanics 3(3) 435-446.

[29] P. Dineva, T. Rangelov (2016) Wave Scattering by Cracks at Macro- and NanoScale in Anisotropic Plane by BIEM. Journal of Theoretical and Applied Mechanics 46(4) 19-35.

[30] X.Q. Fang, J.X. LiU (2011) Dynamic Stress and Electric Displacement around a Nano-Fiber in Piezoelectric Nanocomposites under Electro-Elastic Waves. Philosophical Magazine Letters 91 621-631.

[31] X.Q. Fang, J.X. LiU, L.H. Dou, M.Z. Chen (2012) Dynamic Strength araund Two Interacting Piezoelectric Nano-Fibers with Surfaces/Interfaces in Solid under ElectroElastic Wave. Thin Solid Films 520 3587-3592.

[32] X.Q. FAng, Q. YANG, J.X. LiU, W.J. Feng (2012) Surfaces/Interface Effect around a Piezoelectric Nano-Particle in a Polymer Matrix Under Compressional Waves. Applied Physics Letters 100151602.

[33] T. Rangelov, P. Dineva (2017) Dynamic Fracture Behaviour of a Nanocrack in a Piezoelectric Plane. ZAMM 97(4) 1393-1405.

[34] R.G. Hoagland, M.S. Daw, J.P. HiRTh (1991) Some Aspects of Forces and Fields in Atomic Models of Crack Tips. Journal of Materials Research 6 2565-2571.

[35] B. WANG, J. HAN (2006) Discussion on Electromagnetic Crack Face Boundary Conditions for the Fracture Mechanics of Magneto-Electro-Elastic Materials. Acta Mechanica Sinica 22 233-242.

[36] V.Z. Parton, B.A. Kudryavtsev (1988) "Electromagnetoelasticity". Gordon and Breach Sci. Publ., New York.

[37] A.K. SoH, J.X. LIU (2005) On the Constitutive Equations of Magnitoelectroelastic Solids. Journal of Intelligent Material Systems and Structures 16 597-602.

[38] J. Sladek, V. Sladek, P. Solek, C. Zhang (2010) Fracture Analysis in Continuously Nonhomogeneous Magneto-Electro-Elastic Solids under a Thermal Load by the MLPG. International Journal of Solids and Structures 47 1381-1391. 
[39] L. LI, P. WEI (2014) Surface Wave Speed of Functionally Graded Magneto-ElectroElastic Materials with Initial Stresses. Journal of Theoretical and Applied Mechanics 44(3) 49-64.

[40] J. Luo, X. WANG (2009) On the Anti-Plane Shear of an Elliptic Nano Inhomogeneity. European Journal of Mechanics A/Solids 28 926-934.

[41] J.Y. XU, C.Y. Dong (2016) Surface and Interface Stress Effects on the Interaction of Nano-Inclusions and Nano-Cracks in an Infinite Domain under Anti-Plane Shear. International Journal of Mechanical Sciences 111-112 12-23.

[42] V.B. Shenoy (2005) Atomistic Calculations of Elastic Properties of Metallic fcc Crystal Surfaces. Physical Review B 71(9) 094104. 